\title{
Effectiveness of trivalent seasonal and monovalent influenza A(H1N1)2009 vaccines in population with major chronic conditions of Navarre, Spain: 2010/11 mid-season analysis
}

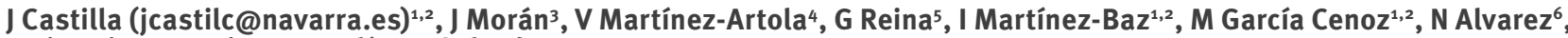 \\ F Irisarri ${ }^{1,2}$, M Arriazu, ${ }^{1,2}$, E Elía ${ }^{3}$, E Salcedo ${ }^{4}$ \\ 1. Instituto de Salud Pública de Navarra (Public Health Institute of Navarre), Pamplona, Spain \\ 2. CIBER de Epidemiología y Salud Pública (Biomedical Research Network of Epidemiology and Public Health) \\ 3. Dirección de Atención Primaria, Servicio Navarro de Salud (Primary Health Care Department), Pamplona, Spain \\ 4. Complejo Hospitalario de Navarra (Navarre Hospital Complex), Pamplona, Spain \\ 5. Clínica Universidad de Navarra (University Clinic of Navarra), Pamplona, Spain \\ 6. Servicio Navarro de Salud (Navarre Health Service), Pamplona, Spain
}

Citation style for this article:

Castilla J, Morán J, Martínez-Artola V, Reina G, Martínez-Baz I, García Cenoz M, Alvarez N, Irisarri F, Arriazu M, Elía F, Salcedo E. Effectiveness of trivalent seasonal and monovalent influenza $\mathrm{A}\left(\mathrm{H}_{1} \mathrm{~N}_{1}\right) 2009$ vaccines in population with major chronic conditions of Navarre, Spain: $2010 / 11$ mid-season analysis. Euro Surveill.

2011;16(7): pii=19799. Available online: http://www.eurosurveillance.org/ViewArticle.aspx?Articleld=19799

Article published on 17 February 2011

We defined a cohort of people with major chronic conditions $(152,585$ subjects) in Navarre, Spain, using electronic records from physicians, to obtain 2010/11 mid-season estimates of influenza vaccine effectiveness. The adjusted estimates of the effectiveness of the 2010/11 trivalent influenza vaccine were $31 \%$ (95\% confidence interval (CI): $20-40 \%$ ) in preventing medically attended influenza-like illness, and $58 \%$ (95\% Cl: $11-80 \%)$ in preventing laboratory-confirmed influenza. Having received the monovalent influenza $A\left(H_{1} N_{1}\right)_{2009}$ vaccine in the $2009 / 10$ season had an independent preventive effect against medically attended influenza-like illness (17\%, 95\% Cl: 1-30\%), and having received both vaccines had $68 \%(95 \% \mathrm{Cl}$ : $23-87 \%)$ effectiveness in preventing laboratory-confirmed influenza.

\section{Introduction}

Because the influenza vaccine composition is adapted every season to the circulating viruses, its effectiveness varies. Estimates of the effectiveness of the vaccine during the influenza season help guiding health interventions aimed at reducing the impact of influenza in the population [1]. In the absence of randomised trials evaluating the efficacy of this vaccine, observational studies are of interest to verify if the expected effect has been achieved [1-3]. A multi-centre European study (I-MOVE: Influenza Monitoring Vaccine Effectiveness in Europe) was launched in 2008, including cohort and case-control studies in several settings. As part of this project, a cohort study is being conducted in Navarre, Spain [1].

During the early 2010/11 season, the influenza $\mathrm{A}\left(\mathrm{H}_{1} \mathrm{~N}_{1}\right)_{2009}$ virus was the predominant circulating influenza virus [4]. It is therefore expected that both the trivalent 2010/11 seasonal vaccine, which includes this virus, [5] and the monovalent influenza $A\left(\mathrm{H}_{1} \mathrm{~N}_{1}\right) 2009$ vaccine [6] may provide some protection. Several studies have reported high effectiveness of the monovalent pandemic vaccine in preventing influenza $A\left(\mathrm{H}_{1} \mathrm{~N}_{1}\right)_{2009}$ during the $2009 / 10$ season [7-11]. The aim of this study was to provide early estimates of the effectiveness of the 2010/11 seasonal vaccine and the influenza $\mathrm{A}\left(\mathrm{H}_{1} \mathrm{~N}_{1}\right) 2009$ vaccine administered during the $2009 / 10$ season in preventing medically attended influenza-like illness (MA-ILI) and laboratory-confirmed influenza during the 2010/11 season. The study was restricted to the population with major chronic conditions, since vaccination with both influenza vaccines was recommended for this group.

\section{Methods}

Study population and data collection

We conducted a prospective cohort study based on electronic records of physicians and laboratories and a nested case-control analysis of swabbed patients in the region of Navarre, Spain. This cohort included all non-institutionalised persons covered by the Regional Health Service (95\% of the population of the region) with known pre-existing major chronic conditions (heart disease, lung disease, renal disease, cancer, diabetes, cirrhosis, dementia, stroke, immunodeficiency and body mass index of 40 or greater). The Navarre Ethical Committee for Medical Research approved the study protocol. The present study analysed the cases registered from 24 October 2010 (first week in which influenza virus was detected in the region) to 22 January 2011.

The seasonal influenza vaccination campaign took place from 11 October to 26 November 2010, although 
a very small number of doses were still administered after that period. The trivalent inactivated non-adjuvanted vaccine (Sanofi Pasteur MSD) was used for all subjects. Monovalent influenza $A\left(\mathrm{H}_{1} \mathrm{~N}_{1}\right)_{2009}$ vaccine had been administered exclusively from November 2009 to January 2010, using the MF59-adjuvanted vaccine from Novartis (Focetria) for children up to the age of 17 years and for adults aged 60 years and older, the ASo3-adjuvanted vaccine from GlaxoSmithKline (Pandemrix) in adults between 18 and 59 years of age, and the non-adjuvanted vaccine from Sanofi Pasteur (Panenza) for pregnant women. All these vaccines were offered free of charge to individuals with major chronic conditions and other populations with specific indications. Precise instructions for registering each dose were given to all vaccination points. For the present study, influenza vaccine status was obtained from the online regional vaccination register that is updated by the healthcare centres of the Regional Health Service. Subjects were considered to be protected 14 days after vaccine administration.

Influenza surveillance is based on automatic reporting of cases from all primary healthcare centres. Cases of MA-ILI are defined according to the International Classification of Primary Care version 2 (code R80) [12]. Two laboratories perform influenza testing in the region and provided the data for virological surveillance. All hospitalised patients with ILI or other acute respiratory diseases were swabbed for influenza virus testing. In addition, through a sentinel network composed of a representative sample of primary healthcare physicians covering $16 \%$ of the population, nasopharyngeal and pharyngeal swabs were taken from all patients

TABLE 1

Population with major chronic conditions included in the cohort study and vaccine coverage by age group, Navarre, Spain, 2010/11 $(n=152,585)$

\begin{tabular}{|l|c|c|c|c|}
\hline Age group & $\begin{array}{c}\text { Population } \\
\text { (number) }\end{array}$ & $\begin{array}{c}\text { Seasonal vaccine 2010/11 } \\
\text { coverage (\%) }\end{array}$ & $\begin{array}{c}\text { Pandemic vaccine in 2009/10 } \\
\text { coverage (\%) }\end{array}$ & $\begin{array}{c}\text { Both vaccines } \\
\text { coverage (\%) }\end{array}$ \\
\hline 1 to 59 years & 81,407 & 11.3 & 7.7 & 4.2 \\
\hline$\geq 60$ years & 71,178 & 60.0 & 26.2 & 22.5 \\
\hline Total & 152,585 & 34.0 & 16.4 & 12.7 \\
\hline
\end{tabular}

\section{FIGURE 1}

Weekly incidence of medically attended influenza-like illness and swabbed patients ( $\mathrm{n}=253)$ according to influenza virus test result in the population with major chronic conditions, Navarre, Spain, 24 October 2010-22 January 2011

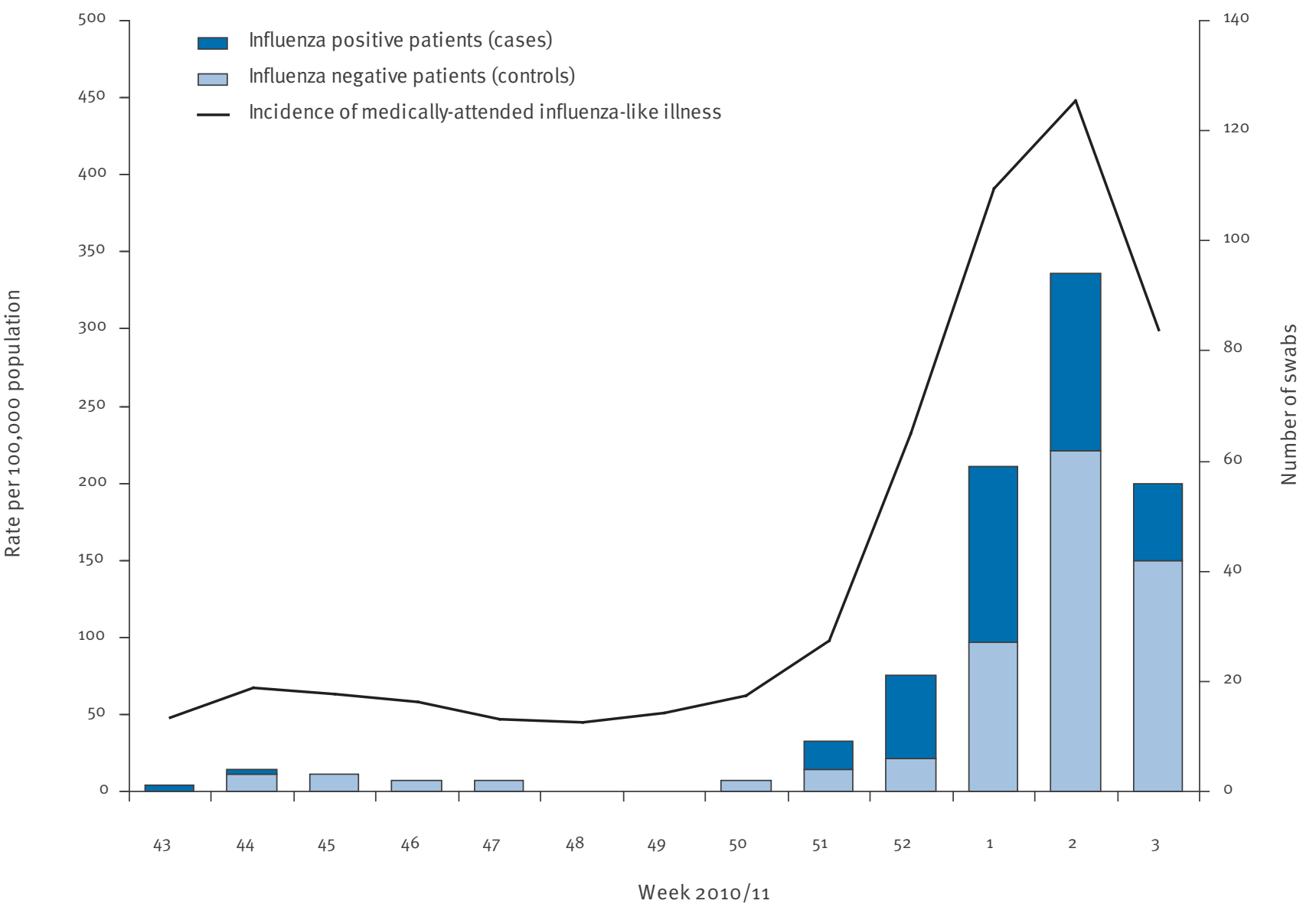


with MA-ILI, after obtaining verbal informed consent. Swabs were processed by RT-PCR assay and virus culture. Positive samples were characterised as influenza $A\left(\mathrm{H}_{1}\right.$ and $\left.\mathrm{H}_{3}\right)$ and $B$ virus using immunofluorescence and RT-PCR. Real-time RT-PCR for detection of the influenza $A\left(\mathrm{H}_{1} \mathrm{~N}_{1}\right)_{2009}$ virus was performed for all swabs.

From the electronic primary healthcare records we obtained the following baseline variables: sex, age, migrant status, district of residence, major chronic con- ditions, number of outpatient visits during the previous 12 months, and children in the household.

\section{Study design and statistical analysis}

In the cohort analysis, the incidence rates of MA-ILI in primary health care were compared in vaccinated and unvaccinated persons. Cox regression models were used to obtain MA-ILI-adjusted hazard ratios (HRs) for influenza vaccination status. Calendar time was used as the underlying time variable, with exit time as the date of MA-ILI diagnosis, death, or 22 January 2011

\section{TABLE 2}

Estimates of the effect of the 2010/11 seasonal influenza vaccine and influenza A(H1N1)2009 vaccine in preventing medically diagnosed influenza-like illness in the population with major chronic conditions, Navarre, Spain, 24 October 2010-22 January $2011(n=152,585)$

\begin{tabular}{|c|c|c|c|c|}
\hline & Person-years & Cases & $\begin{array}{l}\text { Crude hazard ratio } \\
(95 \% \mathrm{Cl})^{\mathrm{a}}\end{array}$ & $\begin{array}{c}\text { Adjusted hazard ratio } \\
(95 \% \mathrm{Cl})^{\mathrm{b}}\end{array}$ \\
\hline \multicolumn{5}{|l|}{ Analysis 1} \\
\hline \multicolumn{5}{|l|}{ Seasonal vaccine $2010 / 11$} \\
\hline Yes & 10,828 & 296 & $0.36(0.32-0.42)$ & $0.69(0.60-0.80)$ \\
\hline No & 26,569 & 1,736 & Reference & Reference \\
\hline \multicolumn{5}{|l|}{ Pandemic vaccine $2009 / 10$} \\
\hline Yes & 6,102 & 172 & $0.78(0.66-0.92)$ & $0.83(0.70-0.99)$ \\
\hline No & 31,295 & 1,860 & Reference & Reference \\
\hline \multicolumn{5}{|l|}{ Analysis 2} \\
\hline Seasonal and pandemic vaccines & 4,108 & 100 & $0.30(0.25-0.37)$ & $0.59(0.47-0.73)$ \\
\hline Only seasonal vaccine $2010 / 11$ & 6,720 & 196 & $0.35(0.30-0.41)$ & $0.69(0.58-0.81)$ \\
\hline Only pandemic vaccine $2009 / 10$ & 1,994 & 72 & $0.72(0.57-0.91)$ & $0.81(0.64-1.03)$ \\
\hline Unvaccinated & 24,575 & 1,664 & Reference & Reference \\
\hline
\end{tabular}

$\mathrm{Cl}$ : confidence interval.

a Cox regression model including vaccination status for 2010/11 seasonal and pandemic influenza $A\left(\mathrm{H}_{1} \mathrm{~N}_{1}\right) 2009$ vaccines.

${ }^{b}$ Cox regression model adjusted for sex, age group, major chronic conditions, outpatient visits during baseline period (tertiles within each age stratum), urban/rural residence, migrant status and children in the household, and stratified by age (1-14; $15-59$; $\geq 60$ years) and health district.

\section{TABLE 3}

Estimates of the effect of the 2010/11 seasonal influenza vaccine and influenza A(H1N1)2009 vaccine in preventing laboratory-confirmed influenza in the population with major chronic conditions, Navarre, Spain, 24 October $2010-22$ January $2011(\mathrm{n}=253)$

\begin{tabular}{|c|c|c|c|}
\hline & Cases/controls & $\begin{array}{l}\text { Crude odds ratio } \\
\qquad(95 \% \mathrm{Cl})^{a}\end{array}$ & $\begin{array}{l}\text { Adjusted odds ratio } \\
(95 \% \mathrm{Cl})^{\mathrm{b}}\end{array}$ \\
\hline \multicolumn{4}{|l|}{ Analysis 1} \\
\hline \multicolumn{4}{|l|}{ Seasonal vaccine $2010 / 11$} \\
\hline Yes & $22 / 78$ & $0.32(0.17-0.60)$ & $0.42(0.20-0.89)$ \\
\hline No & $78 / 75$ & Reference & Reference \\
\hline \multicolumn{4}{|l|}{ Pandemic vaccine $2009 / 10$} \\
\hline Yes & $16 / 51$ & $0.69(0.33-1.41)$ & $0.78(0.35-1.73)$ \\
\hline No & $84 / 102$ & Reference & Reference \\
\hline \multicolumn{4}{|l|}{ Analysis 2} \\
\hline Seasonal and pandemic vaccines & $10 / 43$ & $0.22(0.10-0.47)$ & $0.32(0.13-0.77)$ \\
\hline Only seasonal vaccine $2010 / 11$ & $12 / 35$ & $0.32(0.15-0.67)$ & $0.45(0.19-1.03)$ \\
\hline Only pandemic vaccine $2009 / 10$ & $6 / 8$ & $0.70(0.23-2.12)$ & $0.88(0.25-3.18)$ \\
\hline Unvaccinated & $72 / 67$ & Reference & Reference \\
\hline
\end{tabular}

$\mathrm{Cl}$ : confidence interval.

${ }^{a}$ Logistic regression model including 2010/11 seasonal and pamdemic influenza $A\left(\mathrm{H}_{1} \mathrm{~N}_{1}\right) 2009$ vaccination status.

b Logistic regression analysis adjusted for sex, age $(1-14 ; 15-59 ; \geq 60$ years), children in the household, urban/rural residence, healthcare setting (primary healthcare, emergency room, hospitalisation) and date (Week 43-49 2010; Week 50 2010-Week 1 2011; Week 2-3 2011). 
(end of this mid-season analysis), whichever came first. Vaccination status for the $2010 / 11$ seasonal trivalent inactivated vaccine was included in the analyses as a time-dependent variable. The models were stratified by health district and age (1-14, 15-59, $\geq 60$ years) because patients younger than 15 years are cared for by paediatricians and the vaccine coverage is higher among those aged 60 or older. Other potential confounders were adjusted for in the models, with age in intervals of 10 years and the number of outpatient visits categorised in tertiles within each age stratum.

From the cohort population, all outpatients and hospitalised patients who were swabbed during the study period were included in a case-control analysis that compared seasonal vaccination status in patients in whom any influenza virus was detected (cases) and those who were negative for influenza (controls). Crude and adjusted estimators of the effect were quantified by odds ratios (ORs) with their $95 \%$ confidence intervals $(\mathrm{Cl})$, calculated using logistic regression models.

The effects of the seasonal vaccine and the pandemic influenza $A\left(\mathrm{H}_{1} \mathrm{~N}_{1}\right)_{2009}$ vaccine were evaluated as independent variables in one model, and as a combined variable (unvaccinated, only seasonal vaccine, only pandemic vaccine, or both vaccines) in a differ- ent model. Vaccine effectiveness was estimated as a percentage: $(1-H R) \times 100$ or $(1-O R) \times 100$.

\section{Results}

Vaccine effectiveness in preventing medically attended influenza-like illness

A total of 152,585 persons had major chronic conditions registered at baseline and were included in the cohort study, with $46.6 \%$ aged 60 years old or older. The seasonal influenza vaccine coverage for 2010/11 was $34.0 \%$, and $16.4 \%$ had received the influenza $\mathrm{A}\left(\mathrm{H}_{1} \mathrm{~N}_{1}\right) 2009$ pandemic vaccine in 2009/10 (Table 1).

From week 43 of 2010 (first influenza virus detection in the season) to week 3 of 2011, 2,032 cases of MA-ILI were diagnosed among the 152,585 cohort subjects in primary care centres, with the highest incidence in week 2 of 2011 (Figure 1). Eighty-nine of these patients were swabbed by sentinel physicians, and 51 (57\%) of them were found positive for influenza virus.

The incidence rate was 27 per 1,000 vaccinated person-years with the seasonal vaccine as opposed to 65 per 1,000 unvaccinated person-years ( $p<0.001)$. In the adjusted Cox regression model the seasonal vaccine effectiveness against MA-ILI was $31 \%(H R=0.69$; $95 \% \mathrm{Cl}: 0.60-0.80$ ), and the effectiveness of the monovalent pandemic vaccine was $17 \%(H R=0.83$; $95 \% \mathrm{Cl}: 0.70-0.99)$. As compared with unvaccinated

\section{FIGURE 2}

Effectiveness of the 2010/11 seasonal influenza vaccine in preventing medically attended influenza-like illness and laboratory-confirmed influenza in the population with major chronic conditions, Navarre, Spain ${ }^{\mathrm{a}}$

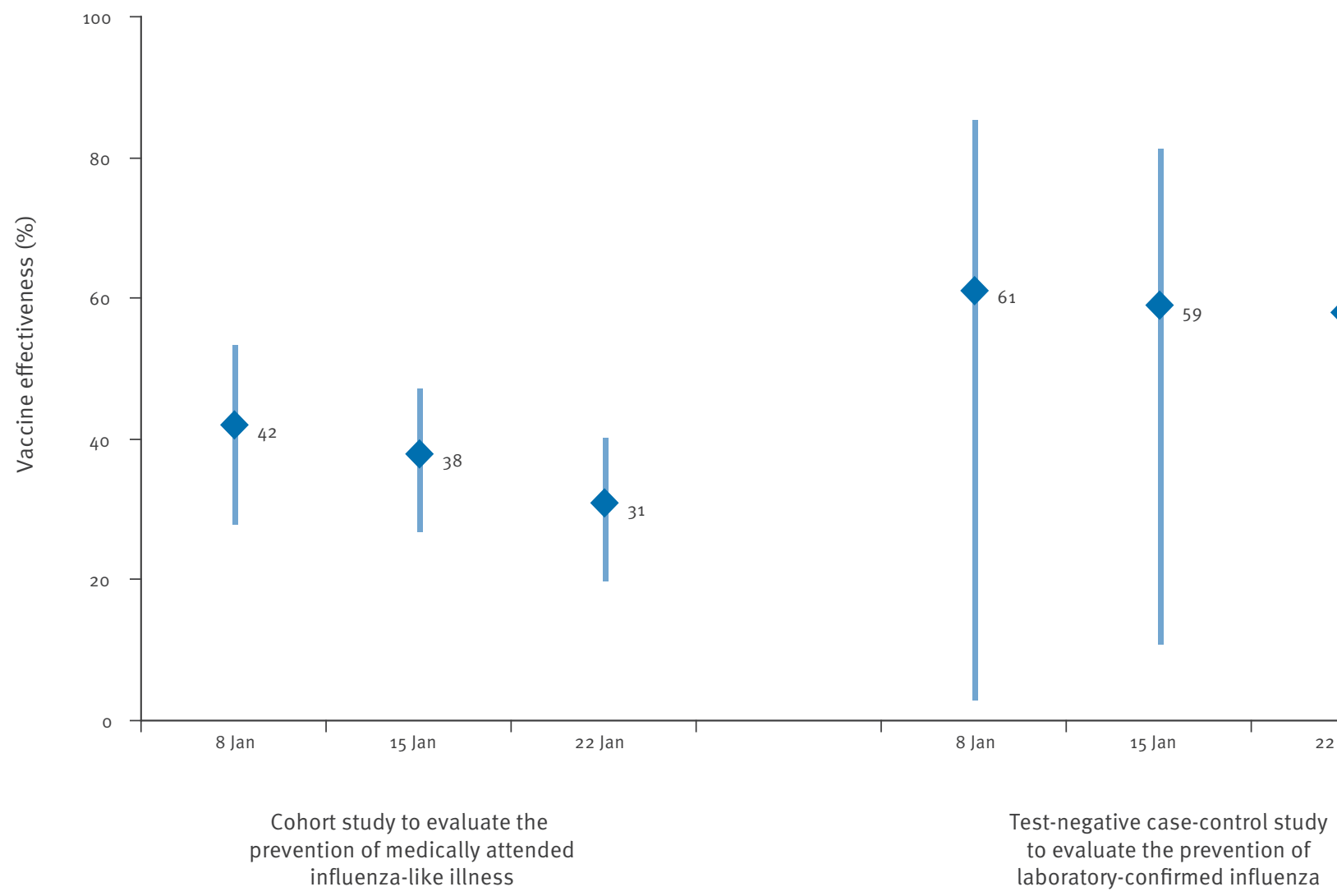

a Preliminary estimates obtained for the periods from 24 October 2010 to 8, 15 and 22 January 2011 , respectively. 
individuals, having received both vaccines provided a $41 \%$ reduction in the incidence of $M A-I L I ~(H R=0.59$; 95\% Cl: 0.47-0.73) (Table 2).

\section{Vaccine effectiveness in preventing}

\section{laboratory-confirmed influenza}

During the study period swabs were analysed from 253 cohort patients who had MA-ILI $(n=89)$ or were treated in hospitals for acute respiratory infection $(n=164)$, and had major chronic conditions (Figure 1). A total of 100 cases (39.5\%) were confirmed for influenza: 97 were positive for the influenza $A\left(\mathrm{H}_{1} \mathrm{~N}_{1}\right) 2009$ virus, one for influenza $A\left(\mathrm{H}_{3} \mathrm{~N}_{2}\right)$ and two for influenza $B$. There were 22 laboratory-confirmed cases in patients who had received the $2010 / 11$ seasonal vaccine. Their mean age was 66 years (range: 52-84 years) and 10 of them had also been vaccinated with monovalent influenza $\mathrm{A}\left(\mathrm{H}_{1} \mathrm{~N}_{1}\right)_{2009}$ vaccine. In the cases with vaccine failure the time from seasonal vaccination to diagnosis ranged 57 to 91 days. At baseline, 10 of these cases had lung diseases, nine had diabetes mellitus, seven had cardiovascular diseases, five had cancers, four had renal diseases and one had liver disease.

Compared with the influenza-negative controls, cases were less likely to have received the influenza seasonal vaccine $(\mathrm{OR}=0.32 ; 95 \% \mathrm{Cl}: 0.17-0.60)$. In the logistic regression analysis adjusting for sex, age (1-14; 15-59; $\geq 60$ years), living with children, living in an urban/rural area, healthcare setting (primary healthcare, emergency room, hospitalisation) and date (Week 43-49 2010; Week 50 2010-Week 1 2011; Week 2-3 2011), seasonal influenza vaccination was associated with a $58 \%$ lower probability of a positive swab $(\mathrm{OR}=0.42 ; 95 \% \mathrm{Cl}$ : $0.20-0.89$ ). The pandemic influenza vaccine showed a lower, not statistically significant, protective effect against laboratory-confirmed influenza $(O R=0.78,95 \%$ $\mathrm{Cl}$ : 0.35-1.73). The interaction term between both vaccines was not significant $(p=0.95)$. Compared with not being vaccinated, having received both vaccines provided $68 \%$ protection against laboratory-confirmed influenza $(\mathrm{OR}=0.32 ; 95 \% \mathrm{Cl}: 0.13-0.77)$ (Table 3).

Early estimates of influenza vaccine effectiveness Effectiveness estimates made at the end of week 1 and 2 of 2011, when the numbers of influenza cases were still increasing, produced similar results (Figure 2). It is worth noticing the progressive decrease in the estimates of effectiveness in preventing MA-ILI, which coincides with a reduction in the percentage of swabs positive for influenza.

\section{Discussion}

The mid-season results of this study show a moderate protective effect of the $2010 / 11$ seasonal influenza vaccine in preventing laboratory-confirmed influenza and MA-ILI during the 2010/11 seasonal period in a high-risk population. In these analyses, receipt of the monovalent influenza $\mathrm{A}\left(\mathrm{H}_{1} \mathrm{~N}_{1}\right) 2009$ pandemic vaccine in the previous season also showed a small preventive effect. Influenza $A\left(\mathrm{H}_{1} \mathrm{~N}_{1}\right)_{2009}$ virus was found in $97 \%$ of the laboratory-confirmed influenza cases and was included in both vaccines, which is consistent with the observed protection. The greatest protective effect was seen in people who had received both vaccines, which could be interpreted as a dose-response effect. Similar findings have been reported in a mid-season analysis in the United Kingdom [13].

This moderate effect is in contrast with the more pronounced protection reported for the $2009 / 10$ season [7-11]. In addition, we detected a number of vaccine failures in persons with laboratory-confirmed influenza. Unlike the pandemic vaccine administered in $2009 / 10$, the $2010 / 11$ seasonal vaccine used in Navarre was not adjuvanted and this could explain a slightly lower immune response. The antigenic drift of the circulating virus could produce a certain degree of mismatch with the vaccine virus, although virological surveillance does not support this so far [14]. Factors such as advanced age or some immunodepression may be more common among people with major chronic conditions, which would explain a poor response to the vaccine. The reduced effect of the monovalent pandemic vaccine in this season can be explained by the loss of immune response more than a year after its administration.

The results presented here are preliminary and may have limited statistical power for some analyses. Therefore the final results for the season may be different. Cohort studies can be affected by biases if those who are vaccinated tend to have poorer health status or if, on the contrary, they tend to take better care of their health than the unvaccinated [15-16], but our analyses were controlled for the most frequently recognised confounders [17]. All the analyses were restricted to the population with major chronic conditions in whom vaccination was indicated. Calendar time was used as the underlying time variable in the Cox regression analysis to control for its possible confounding effect. The case-control analysis only included laboratory-confirmed cases and compared them with controls recruited in the same healthcare settings before patient and physician knew the laboratory result, a fact that reduced selection bias.

The analyses of the vaccine effectiveness against two outcomes, in the same place and period, provide complementary information. The effectiveness of $58 \%$ in preventing laboratory-confirmed influenza can be considered the best estimate of the actual protective effect of the trivalent 2010/11 seasonal vaccine. The effectiveness of $31 \%$ in preventing primary care-attended ILI describes the effect as seen in the clinical practice, where only a part of MA-ILI are confirmed for influenza virus ( $57 \%$ in the study period). That the results obtained using two designs for two different outcomes were consistent reinforces their validity.

Differences between unadjusted and adjusted estimates were greater in the cohort analysis than in the case-control comparison. The test-negative 
case-control analysis provides a better comparability since cases and controls were recruited in the healthcare system under similar circumstances. However, the comparability in the population-based cohort analysis requires a good control of confounding factors.

\section{Conclusion}

Our study shows that it is feasible to provide early estimates of influenza vaccine effectiveness during the season from cohort studies based on healthcare databases. These results support a moderate protective effect of the $2010 / 11$ seasonal vaccine and a low residual effect of last season's monovalent pandemic vaccine against influenza disease in the high-risk population in the $2010 / 11$ season. These results highlight the importance of annual immunisation against influenza of high-risk populations and complementing it with other preventive initiatives such as promotion of basic hygiene measures and avoiding contact with influenza cases.

\section{Acknowledgements}

We are grateful to the Primary Health Care Sentinel Network of Navarra. This work was supported by the I-MOVE (Influenza Monitoring Vaccine Effectiveness in Europe) programme funded by the European Centre for Disease Prevention and Control (ECDC), and by the Carlos III Institute of Health (GRog/0028 and PS09/01179).

\section{References}

1. Valenciano $M$, Ciancio $B C$, Moren $A$, the influenza vaccine effectiveness working group. First steps in the design of a system to monitor vaccine effectiveness during seasonal and pandemic influenza in EU/EEA Member States. Euro Surveill. 2008;13(43):pii=19015. Available online: http://www. eurosurveillance.org/ViewArticle.aspx?Articleld=19015

2. Orenstein WA, Bernier RH, Hinman AR. Assessing vaccine efficacy in the field. Further observations. Epidemiol Rev. 1988;10:212-40.

3. Valenciano M, Kissling E, Ciancio BC, Moren A. Study designs for timely estimation of influenza vaccine effectiveness using European sentinel practitioners networks. Vaccine. 2010;28(46):7381-8.

4. European Centre for Disease Prevention and Control. ECDC risk assessment: Seasonal influenza 2010-2011 in Europe (EU/EEA countries). Stockholm: ECDC; January 2011. Available from: http://www.ecdc.europa.eu/en/ publications/Publications/110125_RA Seasonal Influenza EUEEA_2010-2011.pdf

5. Recommended virus for influenza vaccines for use in the 2010-2011 northern hemisphere influenza. Wkly Epidemiol Rec. 2010;85(10):81-92.

6. World Health Organization. Availability of a candidate reassortant vaccine virus for the novel influenza $A\left(\mathrm{H}_{1} \mathrm{~N}_{1}\right)$ vaccine development. Geneva: WHO; 4 June 2009. Available from: http://www.who.int/csr/resources/publications/ swineflu/ivr153_20090608_en.pdf

7. Wichmann O, Stöcker P, Poggensee G, Altmann D, Walter $D$, Hellenbrand W, et al. Pandemic influenza $A\left(\mathrm{H}_{1} \mathrm{~N}_{1}\right) 2009$ breakthrough infections and estimates of vaccine effectiveness in Germany 2009-2010. Euro Surveill. 2010;15(18): pii=19561. Available from: http://www.eurosurveillance.org/ViewArticle. aspx?Articleld $=19561$

8. Puig-Barberà J, Arnedo-Pena A, Pardo-Serrano F, TiradoBalaguer MD, Pérez-Vilar S, Silvestre-Silvestre E, et al. Effectiveness of seasonal 2008-2009, 2009-2010 and pandemic vaccines, to prevent influenza hospitalizations during the autumn 2009 influenza pandemic wave in Castellón, Spain. A test-negative, hospital-based, case-control study. Vaccine. 2010;28(47):7460-7.
9. Valenciano M, Kissling E, Cohen JM, Oroszi B, Barret AS, Rizzo $C$, et al. Estimates of pandemic influenza vaccine effectiveness in Europe, 2009-2010: results of influenza monitoring vaccine effectiveness in Europe (I-MOVE) multicentre case-control study. PLoS Med. 2011; 8(1):e1000388.

10. Hardelid P, Fleming DM, McMenamin J, Andrews N, Robertson $C$, SebastianPillai P, et al. Effectiveness of pandemic and seasonal influenza vaccine in preventing pandemic influenza $\mathrm{A}\left(\mathrm{H}_{1} \mathrm{~N}_{1}\right) 2009$ infection in England and Scotland 2009-2010. Euro Surveill. 2011;16(2):pii=19763. Available from: http:// www.eurosurveillance.org/ViewArticle.aspx?Articleld $=19763$

11. Wu J, Xu F, Lu L, Lu M, Miao L, Gao T, et al. Safety and effectiveness of a $2009 \mathrm{H}_{1} \mathrm{~N}_{1}$ vaccine in Beijing. N Engl J Med 2010;363(25):2461-23.

12. ICPC-2. International Classification of Primary Care, 2 nd ed. Oxford: Oxford University Press; 1998.

13. Pebody R, Hardelid P, Fleming DM, McMenamin J, Andrews N, Robertson C, et al. Effectiveness of seasonal 2010/11 and pandemic influenza $A\left(\mathrm{H}_{1} \mathrm{~N}_{1}\right) 2009$ vaccines in preventing influenza infection in the United Kingdom: mid-season analysis 2010/11. Euro Survell. 2011;16(6): pii=19791. Available from: http://www.eurosurveillance.org/ViewArticle. aspx?Articleld $=19791$

14. Instituto de Salud Carlos III. Boletín semanal del Sistema de Vigilancia de la Gripe en España. [Weekly Bulletin of the influenza surveillance system in Spain]. № 16, Week 3/2011. Available from: http://vgripe.isciii.es/gripe/ PresentarHomeBoletin.do?boletin $=1 \&$ bol $=308$

15. Simonsen L, Taylor RJ, Viboud C, Miller MA, Jackson LA. Mortality benefits of influenza vaccination in elderly people: an ongoing controversy. Lancet Infect Dis. 2007;7(10):658-66.

16. Jackson LA, Jackson ML, Nelson JC, Neuzil KM, Weiss NS. Evidence of bias in estimates of influenza vaccine effectiveness in seniors. Int J Epidemiol. 2006;35(2):337-44.

17. Nichol KL, Nordin JD, Nelson DB, Mullooly JP, Hak E. Effectiveness of influenza vaccine in the community-dwelling elderly. N Engl J Med. 2007;357(14):1373-81. 